\title{
Divergent streamlines and free vortices in Newtonian fluid flows in microfluidic flow-focusing devices
}

\author{
M. S. N. Oliveira ${ }^{1,2 \dagger}$, F. T. Pinho ${ }^{2}$ and M. A. Alves ${ }^{2}$ \\ ${ }^{1}$ Department of Mechanical and Aerospace Engineering, University of Strathclyde, Glasgow G1 1XJ, UK \\ ${ }^{2}$ Faculdade de Engenharia, Universidade do Porto, Centro de Estudos de Fenómenos de Transporte, Rua \\ Dr Roberto Frias, 4200-465 Porto, Portugal \\ (Received 17 October 2011; revised 4 June 2012; accepted 27 July 2012)
}

The appearance of divergent streamlines and subsequent formation of free vortices in Newtonian fluid flows through microfluidic flow-focusing geometries is discussed in this work. The micro-geometries are shaped like a cross-slot but comprise three entrances and one exit. The divergent flow and subsequent symmetric vortical structures arising near the centreline of the main inlet channel are promoted even under creeping flow conditions, and are observed experimentally and predicted numerically above a critical value of the ratio of inlet velocities $(V R)$. As $V R$ is further increased these free vortices continue to grow until a maximum size is reached due to geometrical constraints. The numerical calculations are in good agreement with the experimental observations and we probe numerically the effects of the geometric parameters and of inertia on the flow patterns. In particular, we observe that the appearance of the central recirculations depends non-monotonically on the relative width of the entrance branches and we show that inertia enhances the appearance of the free vortices. On the contrary, the presence of the walls in three-dimensional geometries has a stabilizing effect for low Reynolds numbers, delaying the onset of these secondary flows to higher $V R$. The linearity of the governing equations for creeping flow of Newtonian fluids was invoked to determine the flow field for any $V R$ as a linear combination of the results of three other independent solutions in the same geometry.

Key words: low-Reynolds-number flows, microfluidics, vortex dynamics

\section{Introduction}

Hydrodynamic focusing at the microscale has been used for various purposes, and has found many interesting practical applications, including micro-mixing, droplet formation, synthesis of micro-particles, among others. The basic design consists of a long micro-channel with three entrances, typically in a cross-like arrangement, as shown in figure 1, in which a central mainstream is shaped by two lateral streams that work as sheath flows. A number of authors have used this type of configuration as passive micro-mixers (Jensen 1998; Knight et al. 1998) and it has been shown that mixing times can be controlled and reduced by adjusting the pressure ratio 
(a)

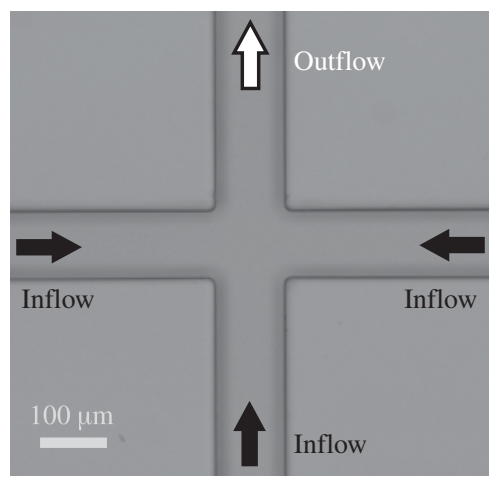

(b)

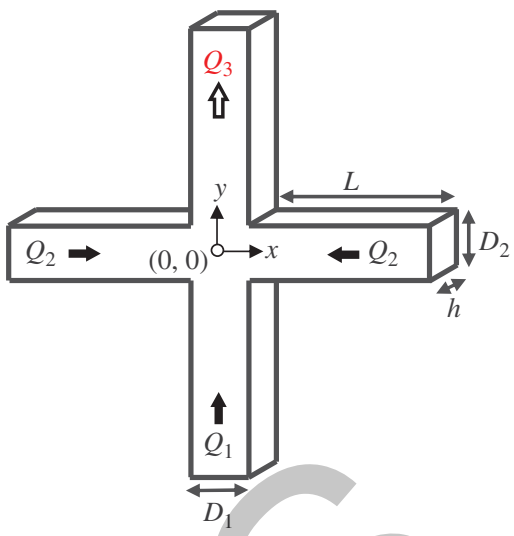

FIGURE 1. The flow-focusing geometry: (a) microscope image showing the central region of the experimental microfluidic channel with three inlets and one outlet; and $(b)$ schematics of the geometry showing the coordinate system and relevant geometrical variables and flow rate, $Q$.

between the main inlet stream and the lateral sheath streams (Jensen 1998). Luo

${ }_{41}$ of droplets with narrow size distribution, whose sizes are tailored by the flow-rate

42 ratio of the two immiscible fluids (Anna, Bontoux \& Stone 2003; Garstecki, Stone \&

43 Whitesides 2005; Anna \& Mayer 2006; Nie et al. 2008). Using the same principle

44 together with a curing or reaction process (usually promoted by UV illumination),

45 solid polymeric particles, including multifunctional particles of controlled shape and

46 size, have also been synthesized (Nisisako, Torii \& Higuchi 2004; Dendukuri \& Doyle 2009).

${ }_{48}$ The strong elongational flows that can be generated at the centre of these devices 49 make them particularly suitable for the study of extensional effects (Arratia, Gollub \& 50 Durian 2008; Oliveira et al. 2009). The effect of elasticity on filament thinning and break-up has been investigated by Arratia et al. (2008) using sets of two immiscible fluids. The authors observed distinct behaviours for Newtonian and polymeric fluids and discussed the suitability of the exponential flow thinning behaviour to measure the extensional viscosity of polymer solutions. Oliveira et al. (2009) investigated numerically the onset of two distinct elastic instabilities in viscoelastic fluid flows using the upper-convective Maxwell (UCM) (Bird, Armstrong \& Hassager 1987) and Phan-Thien-Tanner (PTT) (Phan-Thien \& Tanner 1977) rheological models: one in which the flow becomes asymmetric but remains steady, and a second instability at higher Deborah numbers in which the flow becomes unstable, oscillating periodically in time. This latter type of elastic instability can be exploited to passively promote mixing at the microscale, under low Reynolds number flow conditions. The present authors have previously discussed the possibility of using these geometries with a single fluid to obtain a uniform strain rate for extensional rheometry purposes (Oliveira et al. 2009).

While investigating numerically the effect of operating and geometric parameters on Newtonian fluid flow through flow-focusing devices at low Reynolds numbers, typical of microfluidic flows, we predicted the onset of free vortices upstream of 
the intersection region, located near the centre of the channel and away from any solid-liquid interface. The location at which these vortical structures arise was at first sight unexpected as recirculations typically develop near solid-liquid interfaces for low Reynolds number flows. A well known example in Newtonian fluid flow is the formation of recirculations downstream of an expansion even under creeping flow conditions (Moffatt 1964), which increases in size and intensity as the Reynolds number is increased (Townsend \& Walters 1994; Chiang, Sheu \& Wang 2000; Oliveira 2003; Tsai et al. 2006; Oliveira et al. 2008; Sousa et al. 2011).

The recirculation structures observed near the centreline in the flow-focusing geometry are similar in appearance to bubble-like structures found during vortex breakdown experiments in torsionally driven cavity flows (Leibovich 1978). We note, however, that vortex breakdown occurs in flows with swirl and only at relatively high Reynolds numbers (Escudier 1988), and therefore the underlying mechanism is inherently different from the flow-focusing configuration considered here, even though they may share some flow features. There are various types of vortex breakdown, but essentially the strong swirl creates an adverse pressure gradient in the axial direction, which the axial flow cannot sustain above a critical condition, leading to flow reversal (Lucca-Negro \& O'Doherty 2001).

In this paper we investigate the formation and enhancement of the vortical structures formed at low Reynolds numbers in the flow-focusing geometry and characterize the flow experimentally and numerically. We show that prior to the onset of these central vortices the flow is initially characterized by the appearance of diverging streamlines upstream of the intersection at lower $V R$. We show numerically that such diverging streamlines can occur even in flows without inertia.

The paper is organized as follows. In $\S 2$ we give an overview of the flow-focusing geometry and the experimental set-up. In $\$ 3$, we present a brief outline of the numerical method and computational meshes used. In $\S 4$, an overview of the flow characteristics observed in two-dimensional simulations is presented and in $\S 5$ threedimensional experimental results are discussed and compared to the corresponding three-dimensional numerical simulations. We conclude the paper with a brief summary of our findings in $\S 6$.

\section{Flow geometry and experimental set-up}

We study the flow of a Newtonian fluid (water) through a microfluidic flowfocusing device which presents a cross-like shape and contains three inlets and one outlet channel. The channels used in the experiments were fabricated in polydimethylsiloxane (PDMS) from an SU-8 photoresist mould using standard softlithography techniques (McDonald et al. 2000) which produce planar geometries of constant depth ( $h=100 \mu \mathrm{m}$ in our mould). The geometry is presented in figure 1, where the main variables are identified. The width of the inlet $\left(D_{1}, D_{2}\right)$ and outlet channels $\left(D_{1}\right)$ is kept the same and equal to $100 \mu \mathrm{m}$, which confers a square crosssection to the geometry. The length of the inlet/outlet channels is large enough $\left(L / D_{1}=50\right)$ to guarantee that the flow is fully developed far from the region of interest at all flow rates studied.

A photograph of the experimental set-up is shown in figure 2. A syringe pump with three autonomous modules (neMESYS, Cetoni $\mathrm{GmbH}$ ) was used to inject the fluid in each of the inlet streams independently. In this way, we are able to vary the imposed flow-rate ratio, $F R=Q_{2} / Q_{1}$ (see figure 1), or the corresponding velocity ratio, defined as the ratio of the inlet average velocities in the side streams to the average velocity in

68 


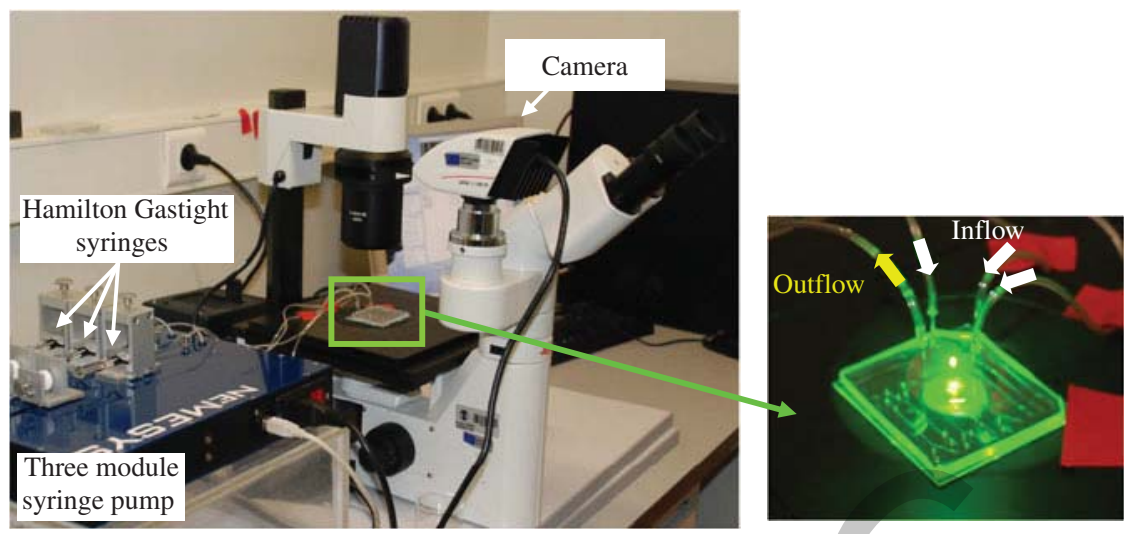

FIGURE 2. (Colour online) Experimental set-up showing the injection system and the optical system used for flow visualization.

the central inlet stream $\left(V R=U_{2} / U_{1}\right)$. Syringes with different volumes $(50 \mu \mathrm{l}-10 \mathrm{ml})$ were used according to the required flow rate and connected to the micro-geometries using Tygon tubing. The same type of tubing was used to connect the outlet channel to a reservoir where the fluid was collected.

Flow visualizations were carried out using streak photography, in which long exposure times are used to capture the flow patterns. For this purpose, the fluid was seeded with $1 \mu \mathrm{m}$ fluorescent tracer particles (Nile Red, Molecular Probes, Invitrogen, Ex/Em: 520/580 nm), and additionally a surfactant (sodium dodecyl sulphate, $0.1 \mathrm{wt} \%$, Sigma-Aldrich) was added to the fluid to reduce the adhesion of fluorescent particles to the channel walls. The optical set-up is composed of an inverted epifluorescence microscope (DMI LED, Leica Microsystems GmbH) fitted with an appropriate filter cube (Leica Microsystems $\mathrm{GmbH}$, excitation BP 530-545 nm, dichroic mirror $565 \mathrm{~nm}$, barrier filter 610-675 nm), a CCD camera (Leica Microsystems GmbH, DFC350 FX), and a 100 W mercury lamp as illumination source.

All images shown in this work were captured at the centre plane using a $10 \times$ $(N A=0.25)$ microscope objective (Leica Microsystems $\mathrm{GmbH})$. For the set-up used, the depth of field corresponds to $\delta z=12 \mu \mathrm{m}$ calculated according to (Meinhart, Wereley \& Gray 2000):

$$
\delta z=\frac{n \lambda_{0}}{(N A)^{2}}+\frac{n e}{(N A) M},
$$

where $n$ is the refractive index, $\lambda_{0}$ is the wavelength of the light (in vacuum), $N A$ is the numerical aperture of the objective, $e$ is the minimum detectable size and $M$ is the total magnification (in this case $e / M=0.65 \mu \mathrm{m}$ ).

Furthermore, a limited number of experiments were carried out using a different technique in which a small amount of Rhodamine B dye (Sigma-Aldrich) was added to the central mainstream while no fluorescent dye (or fluorescent particles) were added to the fluid in the lateral entrances. In this way we take advantage of the relatively large depth of field to highlight the three-dimensionality of the fluid path based on the fluorescence intensity of the imaged dye stream. 


$\begin{array}{llllllllll}W R & 0.1 & 0.3 & 0.5 & 0.8 & 1 & 2 & 3 & 5 & 10 \\ N C & 13965 & 13965 & 16475 & 20491 & 23001 & 35551 & 48603 & 74205 & 85751\end{array}$

TABLE 1. Total number of cells $(N C)$ of the standard two-dimensional computational meshes, with cell size in the central region $\Delta x=\Delta y=D_{1} / 51$.

\section{Numerical method and computational meshes}

In the numerical calculations, an in-house fully implicit finite volume method (FVM) was used to solve the appropriate equations of conservation of mass and momentum assuming the Newtonian fluid flow is isothermal and incompressible (Oliveira, Pinho \& Pinto 1998; Oliveira \& Pinho 1999):

$$
\begin{gathered}
\nabla \cdot \boldsymbol{u}=0, \\
\rho\left(\frac{\partial \boldsymbol{u}}{\partial t}+\nabla \cdot(\boldsymbol{u} \boldsymbol{u})\right)=-\nabla p+\mu \nabla^{2} \boldsymbol{u},
\end{gathered}
$$

where $\rho$ is the density of the fluid, $t$ the time, $\boldsymbol{u}$ the velocity vector, $p$ the pressure and $\mu$ the dynamic viscosity.

We use an implicit first-order Euler scheme for time discretization, central differences for the discretization of the diffusive terms and the CUBISTA highresolution scheme (Alves, Oliveira \& Pinho 2003) for the discretization of the advective terms of the momentum equation. The details of the numerical method will not be repeated here as they have been described thoroughly elsewhere (Oliveira et al. 1998; Oliveira \& Pinho 1999; Alves et al. 2003). A large number of simulations were done under creeping flow conditions, i.e. in the limit when $R e \rightarrow 0$. To simulate such flows, we neglect the advective term on the left-hand side of the momentum equation (3.2), hence solving the corresponding Stokes flow. In those cases we keep the transient term $(\rho \partial \boldsymbol{u} / \partial t)$ and use a pseudo-time-marching algorithm to achieve steady flow conditions. When steady state is achieved the transient term vanishes, and we recover the Stokes equation, valid for creeping flow.

We perform both two- and three-dimensional simulations in which the standard meshes are block-structured and divide the central region of the geometry uniformly into control volumes of size $\Delta x=\Delta y=D_{1} / 51$. In the three-dimensional simulations, the size and shape of the geometry were kept equal to the experiments, with side streams being introduced into the central mainstream through channels of equal dimensions. In this case two sets of meshes were used: a standard mesh used to perform most of the calculations $\left(N C=140625, \Delta x=\Delta y=\Delta z=D_{1} / 25\right)$ and a more refined mesh having nearly twice the number of cells in each direction $\left(N C=1125000, \Delta x=\Delta y=\Delta z=D_{1} / 51\right)$. In two-dimensional simulations, other configurations have also been tested: in particular, the width of the lateral channels $\left(D_{2}\right)$ was varied, while the width of the outlet channel was kept equal to $D_{1}$. The relative width of the entrance branches $\left(W R=D_{2} / D_{1}\right)$ accounts for the effects of geometric parameters and was varied from 0.1 to 10 . Mesh refinement tests have been carried out elsewhere (Oliveira et al. 2009) demonstrating the good accuracy of the calculations using this mesh $\left(\Delta x=\Delta y=D_{1} / 51\right)$. The total number of cells $(N C)$ varies according to the specific geometric configuration under consideration, as shown in table 1. 
(a)

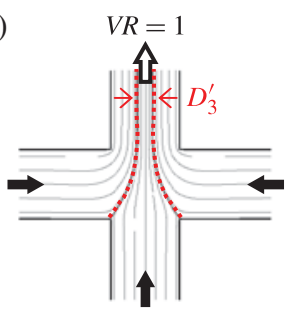

(e)

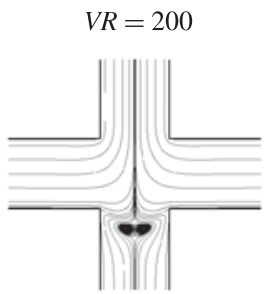

(b)

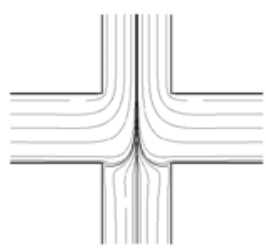

$(f)$

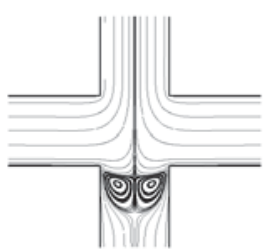

(c)

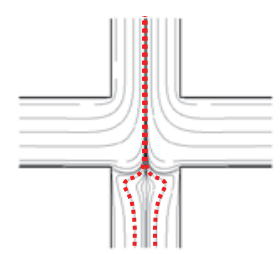

$(g)$

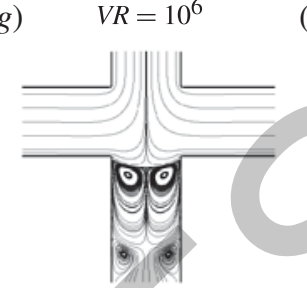

$(d)$

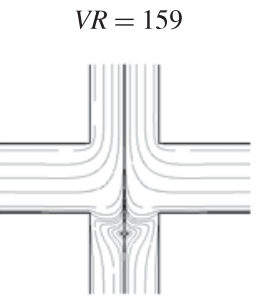

(h) $\quad V R \rightarrow \infty$

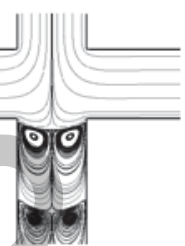

FIGURE 3. (Colour online) Effect of $V R$ on the flow patterns obtained numerically under creeping flow through a 2D flow-focusing geometry with $W R=1:(a) V R=1 ;(b) V R=20$; (c) $V R=100 ;(d) V R=159 ;(e) V R=200 ;(f) V R=1000 ;(g) V R=10^{6} ;(h) V R \rightarrow \infty$. For $V R=1(a)$ the dashed lines highlight the separation streamlines that define the converging region. For $V R=100(c)$ the dashed lines highlight the diverging character of the streamlines upstream of the central region.

The physical properties of the fluid used in the three-dimensional numerical calculations were selected to match those measured experimentally, i.e. the viscosity was set to $0.891 \mathrm{mPa} \mathrm{s}$ and the density to $997 \mathrm{~kg} \mathrm{~m}^{-3}$, corresponding to water at $25^{\circ} \mathrm{C}$, the temperature at which the experiments were performed.

\section{Two-dimensional results}

In this section we present an overview of the flow field obtained using numerical calculations considering a simplified two-dimensional flow-focusing geometry.

\subsection{Creeping flow characteristics}

The flow patterns obtained using two-dimensional (2D) numerical calculations under creeping flow conditions $(R e=0)$ are shown in figure 3. Under these particular operational conditions, the flow is steady and symmetric about the plane $x=0$ and the two opposing lateral fluid streams shape the central mainstream that is flowing perpendicularly to the lateral entrances, generating a converging flow region. For $V R=1$ (figure $3 a$ ), we use (red) dashed lines to show the separation streamlines that define the border between the flow entering from the lateral arms and the flow coming from the central inlet. The converging flow region, delimited by the separation streamlines, can be visualized as a smooth contraction geometry in which there is slip at the walls. In fact for low $V R$, the separation streamlines define a nearly hyperbolic shaped contraction, which is known to generate strong extensional flows near the centreline with nearly constant strain rate (James, Chandler \& Armour 1990; Oliveira et al. 2007; Campo-Deaño et al. 2011).

As $V R$ is increased, the curvature of the separation streamlines near the lateral entrances is enhanced, and the Hencky strain imposed in the converging region increases. Here the Hencky strain is defined based on the widths of the converging 


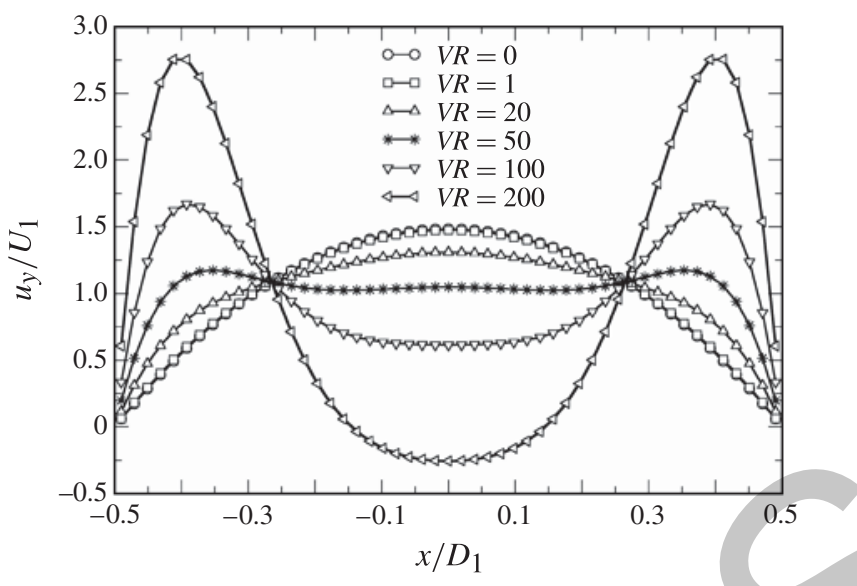

FIgURE 4. Axial velocity profiles at $y / D_{1}=-0.75$ for various $V R$ under creeping flow conditions (2D flow, $W R=1$ ).

region upstream and downstream of the central region, $\varepsilon_{H}=\ln \left(D_{1} / D_{3}^{\prime}\right)$ where $D_{3}^{\prime}$ is shown in figure $3(a)(V R=1)$. For high strains, when the assumption $U_{3}^{\prime} \approx 1.5 U_{3}$ is a reasonable approximation, the Hencky strain can be expressed as $\varepsilon_{H}=\ln [3(1+2 F R) / 2]$. Simultaneously, the streamlines in the mainstream channel just upstream of the central region (for $-1 \lesssim y / D_{1} \lesssim-0.75$ ) become increasingly divergent (see the red dashed streamlines in figure $3 c$ for $V R=100$ ), i.e. the flow moves first towards the wall and then converges again toward the contraction, as if avoiding an invisible obstacle (Alves \& Poole 2007). Streamlines with this characteristic shape are commonly observed in contraction flows with viscoelastic fluids with high extensional viscosity, but not with Newtonian fluids under similar flow conditions (Evans \& Walters 1989; Boger \& Binnington 1990; Hulsen 1993; Rodd et al. 2005; Alves \& Poole 2007). Diverging flow is generally ascribed to fluid elasticity in strong extensional fields, and inertia- and deformation-rate-dependent material functions were seen to enhance its intensity (Rodd et al. 2005). However, Alves \& Poole (2007) have shown numerically that inertia and shear-thinning conditions are not required to observe divergent streamlines for contraction flows of viscoelastic fluids. Here, we show that diverging streamlines can also be observed with Newtonian fluids in the flow-focusing device under creeping flow conditions, i.e. without inertia or elasticity. In other words, we demonstrate that divergent streamlines can arise due to the coupling of geometric and viscous effects alone.

The evolution of the divergent flow in the central inlet arm can be followed in more detail by analysing the velocity and pressure profiles in that region. Figure 4 shows the axial velocity profiles along the $x$-direction in the region of the divergent streamlines, specifically for $y / D=-0.75$. For $V R=1$, the profile has a quasi-parabolic shape, but as $V R$ increases, the velocity at the centreline decreases, while an increase is seen close to the bounding walls. Eventually, the maximum axial velocity shifts from the centreline and the profiles exhibit strong velocity overshoots close to the sidewalls. Further, the axial velocity profiles along the $x=0$ centreline shown in figure 5(a) exhibit an undershoot just upstream of the converging region, which is a fingerprint of divergent flow (Alves \& Poole 2007). The magnitude of this undershoot increases gradually with increasing $V R$. Furthermore, as shown in figure $5(b)$, along 

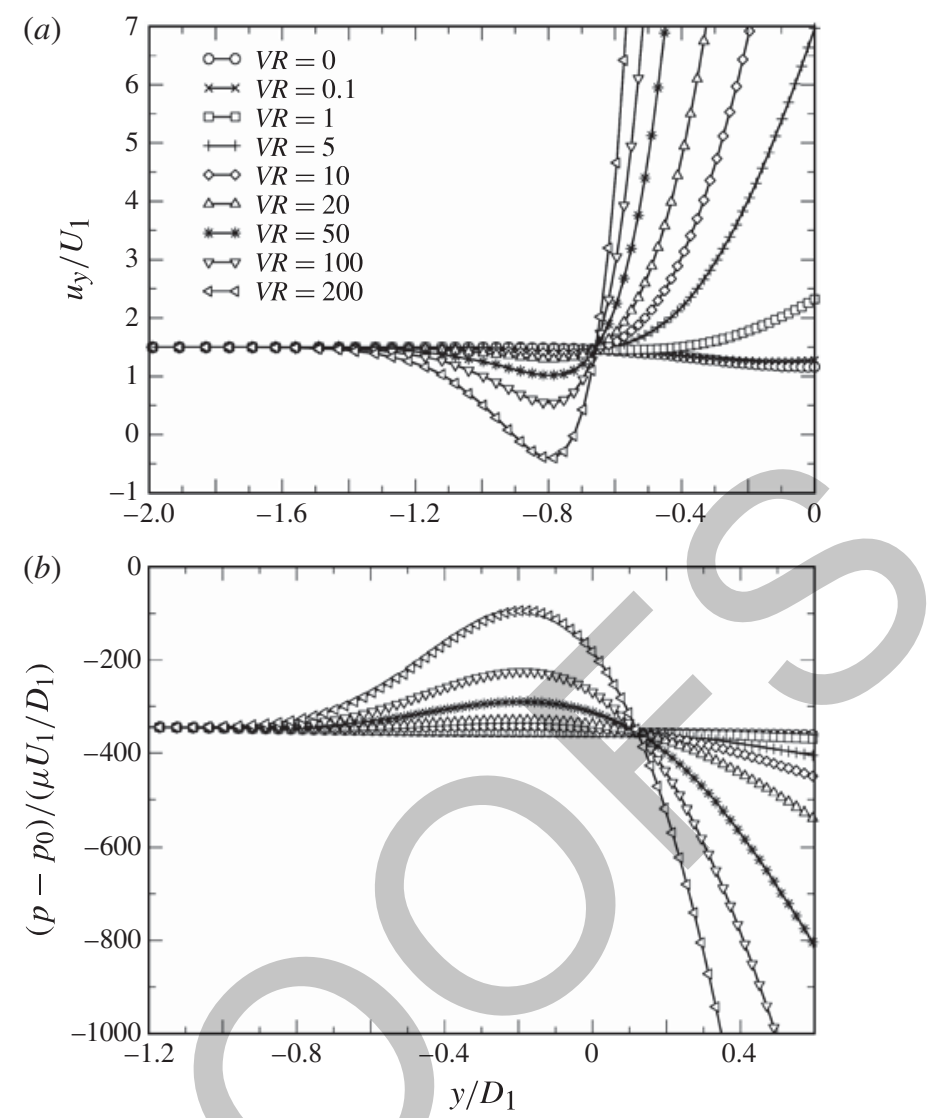

FIgURE 5. (a) Axial velocity and (b) pressure profiles along the centreline $\left(x / D_{1}=0\right)$ for various $V R$ under creeping flow conditions (2D flow, $W R=1$ ).

the centreline adverse pressure gradients are generated just upstream of the centre of the device to match the pressures resulting from the central and sheath inflows which eventually lead to flow separation.

It is interesting to note that all the profiles at different $V R$ (in figures 4 and 5) intersect each other at the same location, suggesting that these may belong to the same family of curves. In fact this is further supported by a theoretical analysis of the governing equations. It is well known that for two-dimensional creeping flows, the stream function for an incompressible Newtonian fluid flow satisfies the biharmonic equation

$$
\nabla^{4} \psi=0,
$$

where $\psi$ is the stream function $(u=\partial \psi / \partial y ; v=-\partial \psi / \partial x)$. Such creeping flow solutions can be combined linearly, with the end result itself being a solution to the Stokes equation

$$
a \nabla^{4} \psi_{1}+b \nabla^{4} \psi_{2}=\nabla^{4}\left(a \psi_{1}+b \psi_{2}\right)=\nabla^{4} \psi^{*}=0,
$$

where $a$ and $b$ are arbitrary scalars. This characteristic, which ensues from the linear nature of the governing equations in creeping flows in conjunction with linearity and 


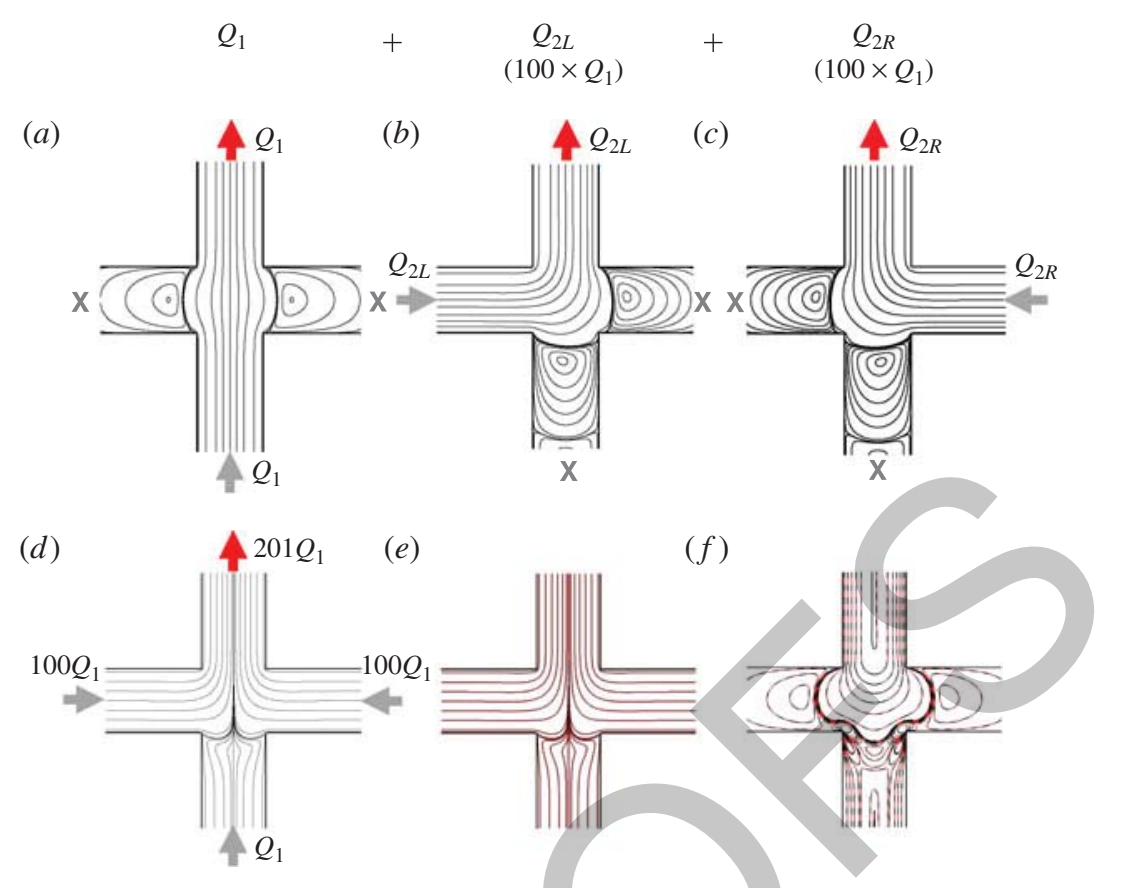

FIGURE 6. (Colour online) Linear combination of three reference flow fields $(a-c)$ to determine the streamlines for $(d) V R=100$. $(e, f)$ Comparison of the flow fields for $V R=100$ (2D flow, $W R=1$ ) obtained by a direct numerical simulation (red lines) and by linear combination of reference solutions (black lines): $(e)$ streamlines; $(f)$ normalized axial velocity contour lines.

symmetry in the boundary conditions, is very useful in deriving results for a given flow without solving it fully. It should be highlighted, however, that any predictive capabilities are limited to the accuracy of the original reference results, i.e. the results are still mesh-dependent, which means that any inaccuracies in the base simulations will be reflected in the resulting predictions.

A consequence of the above analysis is that by using any three linearly independent cases we are able to predict the flow field in the cross-geometry for any other $V R$ in the same mesh and, in fact, for any other flow configuration, whether symmetric or not. We have tested this approach using the three reference cases illustrated in figure $6(a-c)$. Figure 6(e,f) shows a comparison between the results obtained numerically for $V R=100$ and those calculated by combining the three flow fields, considering $Q_{2 L} / Q_{1}=Q_{2 R} / Q_{1}=100$. It is clear that exactly the same solution is obtained independently of the method used, both in terms of the streamlines and in terms of the normalized velocity field (where any inconsistencies would be easily detected). Furthermore, we are able to predict the flow in any type of crosslike geometry with four inlets/outlets (whether in a flow-focusing or a cross-slot arrangement).

Given this characteristic of the flow, we are able to collapse the corresponding velocity profiles for different $V R$ onto a single master curve using an appropriate normalization. The same can be done for other variables such as pressure, normal stress differences, etc. Figure 7 shows the profiles of the axial velocity, pressure and the first normal stress difference normalized using the reference case $(V R=0)$. It is 

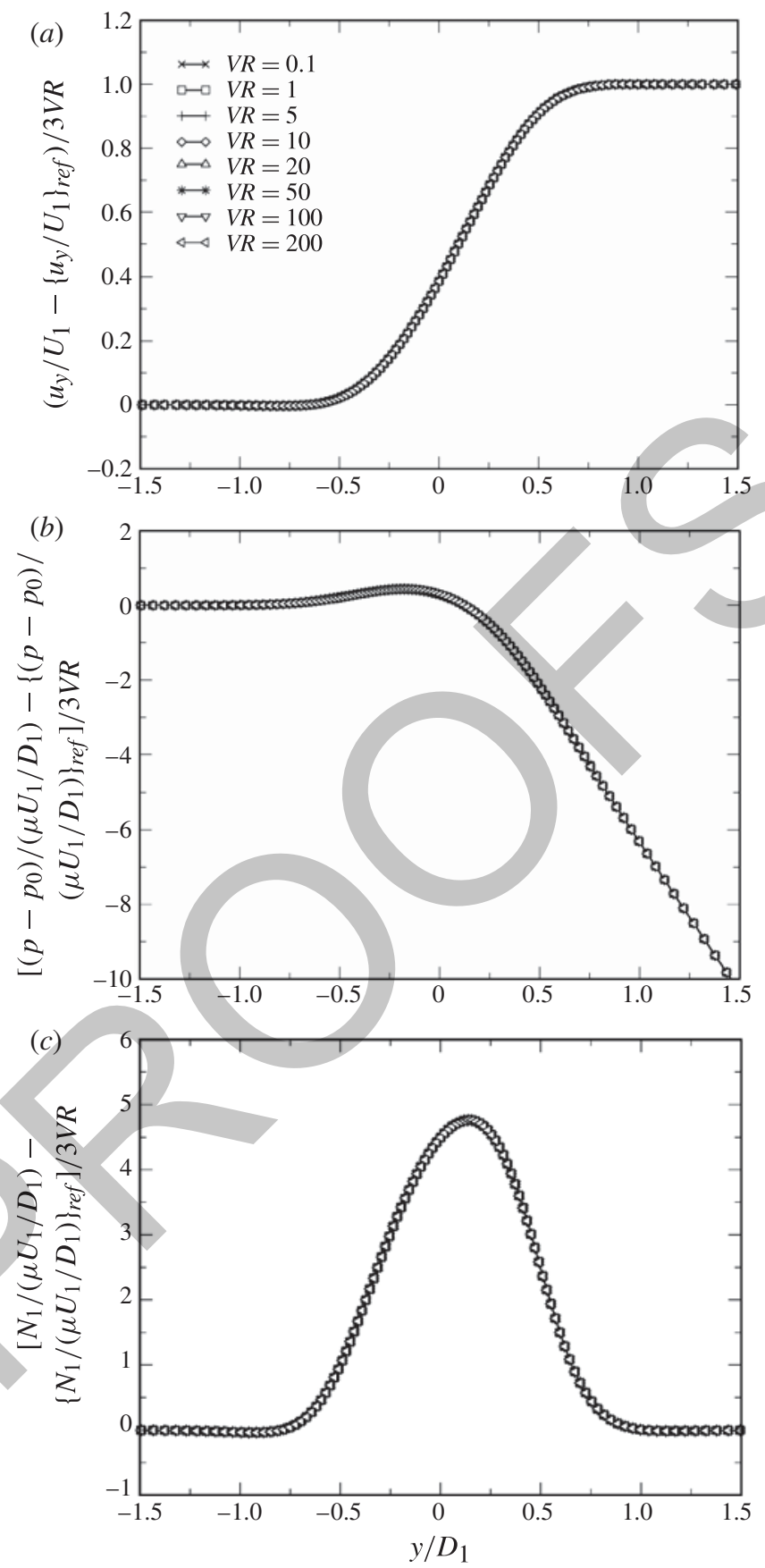

FIGURE 7. (a) Normalized axial velocity, $(b)$ pressure and $(c)$ first normal stress difference $\left(N_{1}=\tau_{y y}-\tau_{x x}\right)$ profiles along the centreline $\left(x / D_{1}=0\right)$ for various $V R$ under creeping flow conditions ( $2 \mathrm{D}$ flow, $W R=1$ ). The reference conditions (ref) were taken as those for $V R=0$. master curve, and coefficient 3 is used for convenience as it ensures a normalization of ${ }_{278}$ the master velocity profile between 0 and 1 . 
(a)

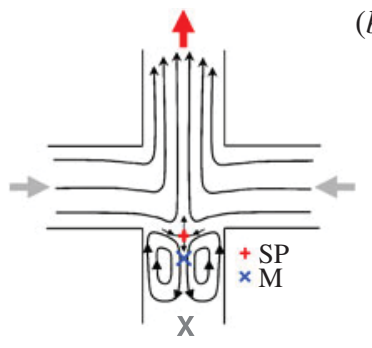

(b)

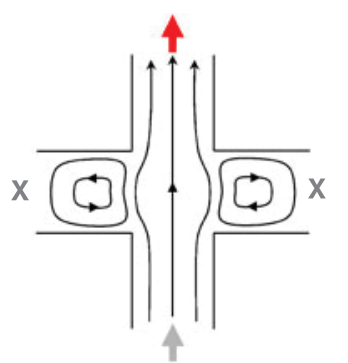

(c)
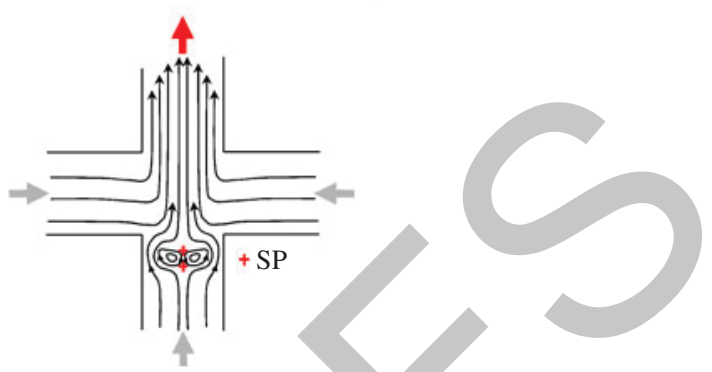

FIGURE 8. (Colour online) Mechanism of formation of free vortices showing the two independent base flows. (a) Flow generated by the collision between two opposed streams equivalent to a T-channel with a long cavity. A free stagnation point, SP (marked with a red ' + '), is generated at the point of collision between the two streams, and recirculations form below this SP yielding a minimum (negative) velocity close to point $\mathrm{M}$ (marked with a blue ' $\times$ '). (b) Flow entering from the lower channel and exiting through the upper channel. When the negative velocities in the lower channel of flow $(a)$ are greater (in magnitude) than the positive velocities of flow $(b)$, then flow $(c)$ is generated, corresponding to our results with flow-focusing configuration above a critical $V R$.

\subsection{Onset and development of free central vortices} below the SP. Nevertheless, if the contribution of flow $(b)$ is much lower than that of flow (a) (i.e. at high $V R$ ), there will still be a region of negative velocities at the $x=0$ 
centreline. Conversely, near the walls of the lower inlet channel, the fluid is always moving upwards, since both contributing flows exhibit positive velocities in this region. These two facts necessarily imply the formation of two symmetrical recirculations near the $x=0$ axis, rather than close to the walls as sketched in figure $8(c)$. If, on the other hand, $V R$ is low, the contribution of flow $(b)$ is sufficient to ensure that the velocities in the $x=0$ line are always positive, thus suppressing the vortex formation. Consequently, there will exist a critical point along the centreline at which the two contributions cancel out, i.e. the minimum velocity along the centreline is precisely zero, marking the onset of vortex formation. This critical point may be estimated from a linear combination of flows $(a)$ and $(b)$ by computing the value of $V R$ for which the minimum centreline velocity reaches zero. This estimate yields a value for $V R_{c}$ in excellent agreement with the onset of vortex formation observed in figure 3 . The onset of flow separation can also be intuitively thought to be due to the locally high pressure generated at the intersection of the lateral streams. This adverse pressure gradient experienced by the fluid elements emerging from the lower channel will lead to flow separation when the adverse pressure gradient is sufficiently high, a phenomenon that increases with an increase in $V R$.

\subsection{Effect of the velocity ratio (VR)}

As described above, for sufficiently large velocity ratios, the minimum velocity at the centreline reaches zero (for $V R \approx 159$ ). Beyond this $V R$ value, a reversal of the axial velocity along the centreline is observed, as shown by the negative axial velocities $U_{y}$ in figure $5(a)$ (e.g. for $V R=200$ ), leading to the formation of a pair of symmetrical recirculations near the centreline of the mainstream inlet channel just upstream of the central region (see figure $3 d, V R=159$ ), at the core of the diverging streamline region. Recirculation growth is observed as $V R$ is further increased until a second recirculation of lower intensity starts to form (see figure $3 g, V R=10^{6}$ ), but now attached to the wall. In fact, for $V R \rightarrow \infty$ the central inlet channel acts as a deep cavity with no net flow $\left(Q_{1}=0\right)$ (as sketched in figure $8 a$ ), and in this case a trail of recirculations is observed all along the cavity (see figure $3 h, V R \rightarrow \infty$ ), with each subsequent pair of recirculations driven by the previous pair. The decay of the intensity of this chain of recirculations can be predicted according to the theoretical analysis of Moffatt (1964).

This sequence of recirculations is analogous to that observed when a true cross-slot geometry is considered (two entrances and two exits) with unbalanced outflow streams. In this case, depicted in figure $9(b)$, vortical structures are also formed as $V R$ increases, and they appear at nearly the same value of $V R$ as for the flow-focusing geometry of figure $9(a)$. However, no divergent flow is observed in the cross-slot, and the primary recirculations are now localized at the wall rather than at the centre of the channel.

We should emphasize that we are also able to predict the onset of vortex formation for any of these configurations by applying the linear combination of base solutions described in $\S 4.1$. By using reference solutions $V R=0$ and $V R \rightarrow \infty$, one can predict the Newtonian fluid flow fields for both a flow-focusing arrangement (parameters $a$ and $b$ in (4.2) are both positive scalars) and a cross-slot geometry (parameters $a$ and $b$ have opposite signs).

In figure 10, we show the effect of $V R$ on the size of the primary recirculations, $y_{R}$, of the flow focusing device. The size was determined at the centreline as also illustrated in figure 10. Above the critical $V R$, the centreline velocity becomes negative, leading to the formation of free central vortices, which grow in size, as shown qualitatively in figure 3 and quantitatively in figure 10. Initially, this growth follows closely a square-root function, $y_{R} / D_{1}=a_{1} \sqrt{\ln (V R)-\ln \left(V R_{c}\right)}$, where $a_{1}$ is a fitting 
(a)

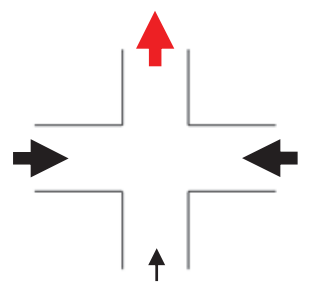

(b)

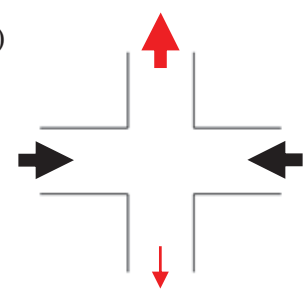

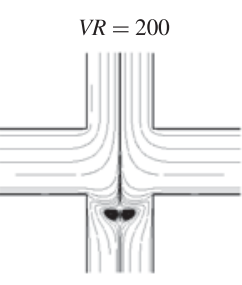

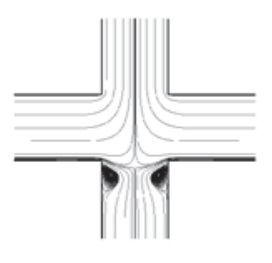

$V R=1000$

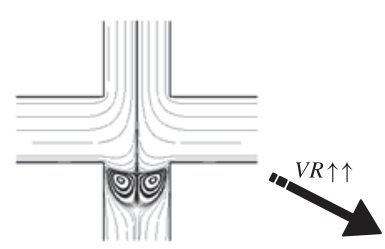

$V R \rightarrow \infty$
$\|L\||\|| \mid$
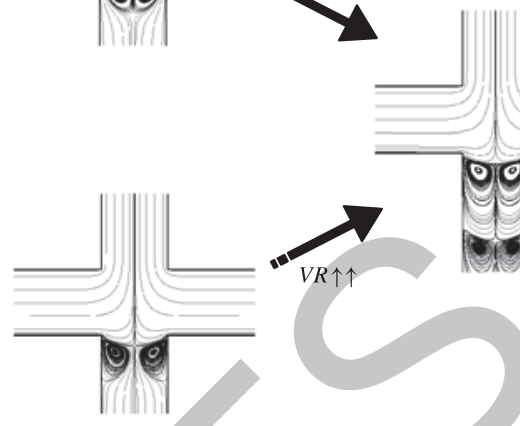

FIGURE 9. (Colour online) Vortex formation under creeping flow conditions (2D flow, $W R=1)$ : comparison between $(a)$ the flow-focusing device and $(b)$ the cross-slot geometry with unbalanced outflow streams.

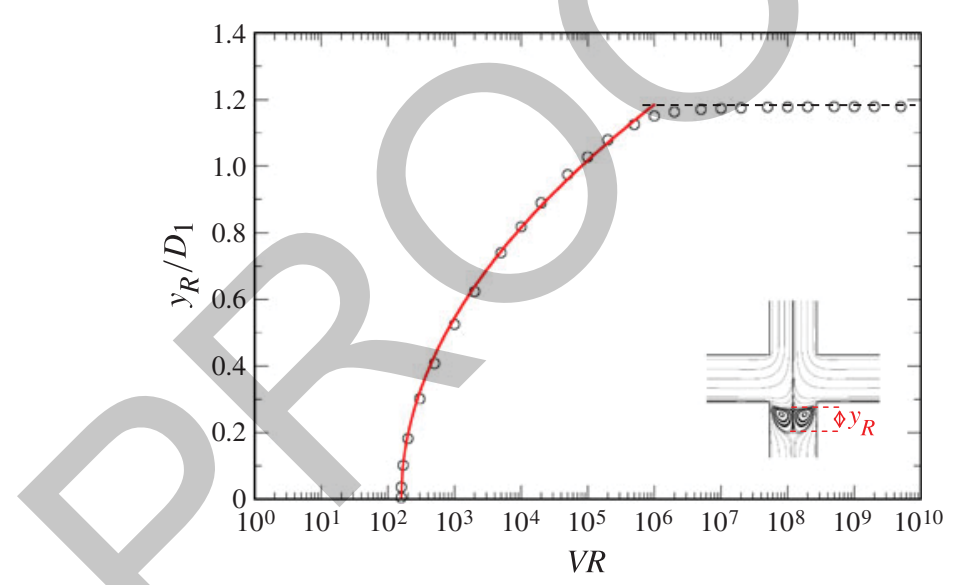

FIGURE 10. (Colour online) Effect of $V R$ on vortex size under creeping flow conditions (2D flow, $W R=1$ ). The symbols represent the numerical data, the black dashed line indicates the asymptotic value at $V R \rightarrow \infty$ and the red solid line corresponds to the function $y_{R} / D_{1}=a_{1} \sqrt{\ln (V R)-\ln \left(V R_{c}\right)}$, with $a_{1}=0.61$ and $V R_{c}=159$.

parameter, and then approaches asymptotically the maximum vortex size reached for $V R \rightarrow \infty$. The value of $V R$ at which the vortex practically reaches its maximum size $\left(\sim 0.9 y_{\max }\right)$ coincides with the onset of the second set of recirculations.

\subsection{Effect of the width ratio (WR)}

The effect of the width ratio was also examined numerically using two-dimensional calculations under creeping flow conditions. In figure 11, we show the flow patterns for two distinct width ratios: $W R=0.3$ and 3 .$$
51
$$

352

353

354 
(a)
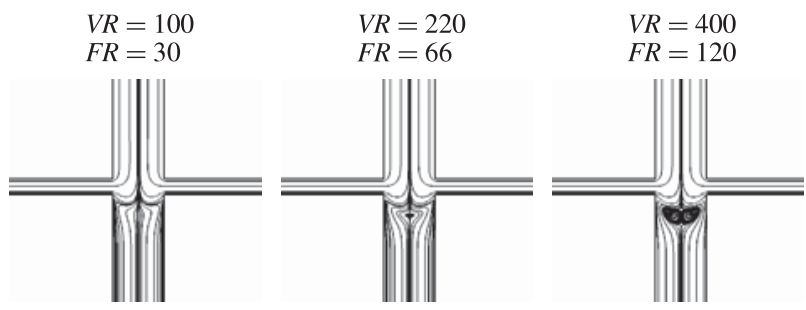

(b)
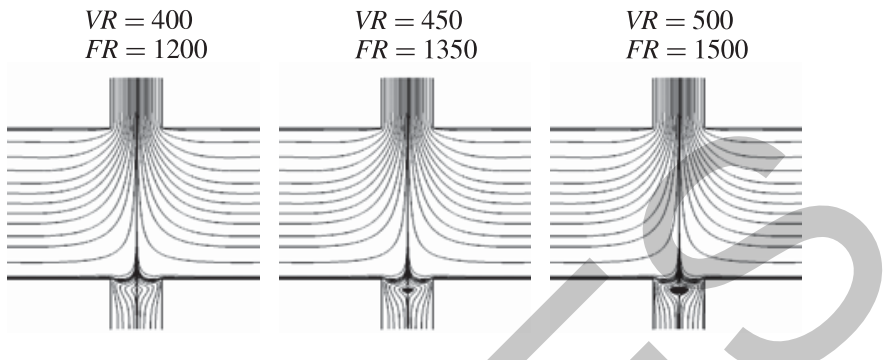

FIGURE 11. Flow patterns obtained numerically under creeping flow through a 2D flow-focusing geometry with (a) $W R=0.3$ and $(b) W R=3$.

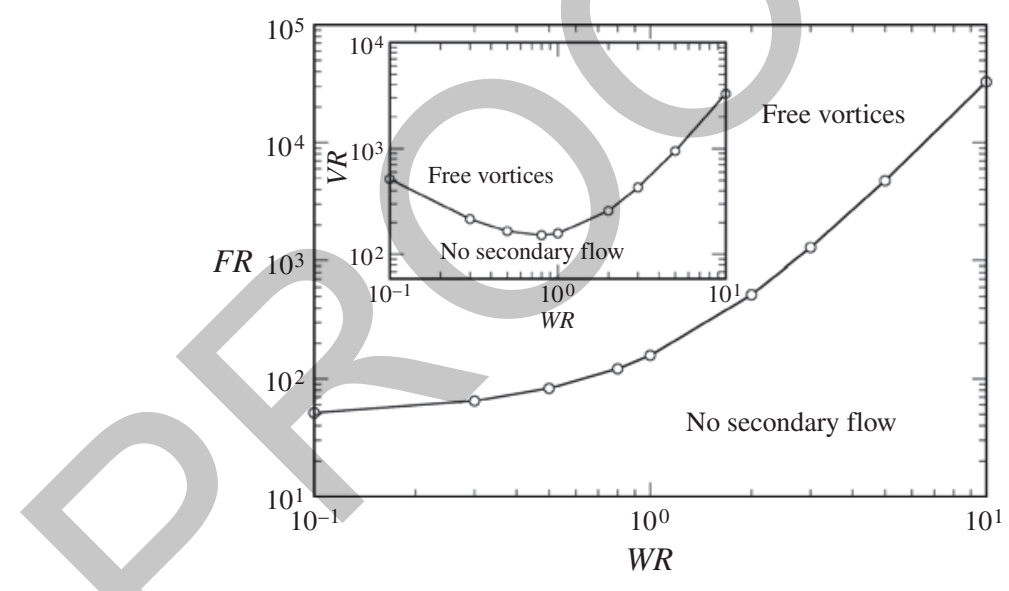

FIGURE 12. Effect of $W R$ on the critical flow-rate ratio and on the critical velocity ratio (inset) for the onset of secondary flow (2D flow, $R e=0$ ).

The behaviour observed for $0.1 \leqslant W R \leqslant 10$ is qualitatively similar to that observed for $W R=1$, i.e. as $V R$ increases, the divergent character of the streamlines is enhanced, followed by the formation and subsequent growth of central recirculations. However, the critical value of $V R$ for the onset of the central recirculations depends on $W R$, as shown in the inset of figure 12 . The transition envelope exhibits non-monotonic behaviour with the minimum value of the critical $V R$ attained for $W R \approx 0.8$. However, in terms of flow-rate ratio (remember that $F R=V R \times W R$ ), the variation of its critical value with $W R$ is monotonic (see figure 12).

The vortex size was also measured as a function of $V R$ for different values of $W R$, which are shown in the inset of figure 13. All the curves show the same shape, and 


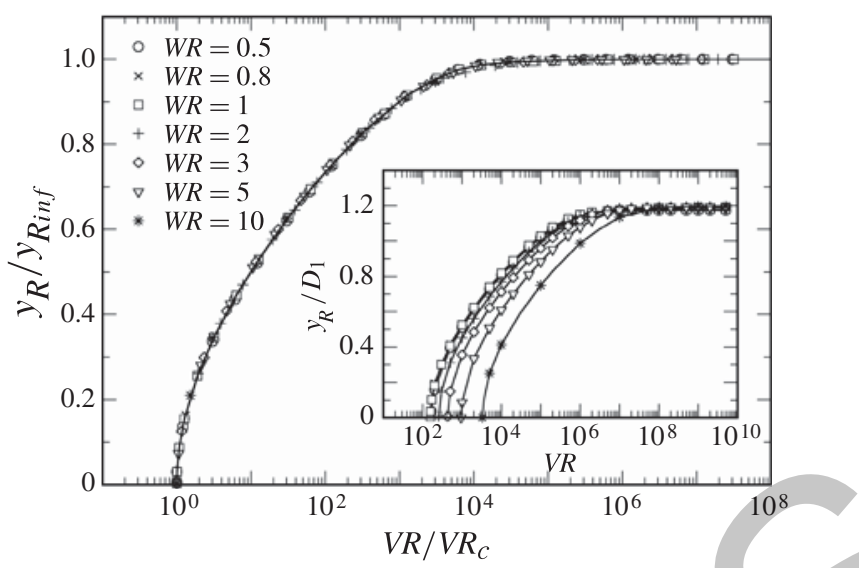

FIGURE 13. Vortex size under creeping flow conditions for various $W R(2 \mathrm{D}$ flow, $W R=1)$.

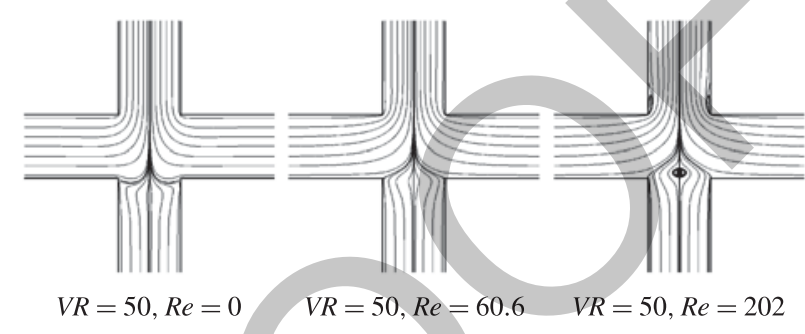

FIGURE 14. Effect of Re on the flow patterns through a 2D flow-focusing geometry with $V R=50(W R=1)$.

tend to maximum vortex sizes reached at $V R \rightarrow \infty\left(y_{R i n f}\right)$, which is almost independent of $W R$. Once again, all the curves can be collapsed onto a master curve by appropriate normalization, as shown in the main plot of figure 13, in which the vortex size is normalized using $y_{\text {Rinf }}$ and $V R$ is normalized by the critical velocity ratio for the onset of vortex formation.

\subsection{Effect of inertia}

The effect of inertia on the development of secondary flow was analysed at a constant width ratio $(W R=1)$. To account for inertial effects, we define the Reynolds number in terms of the exit channel quantities: $R e=\rho U_{3} D_{3} / \mu$.

In figure 14, we show the effect of $R e$ on the flow patterns for $V R=50$, which did not exhibit free vortices under creeping flow conditions. Inertia pushes the divergent streamline region forward into the centre of the flow-focusing device and promotes the onset of central vortices, which appear at $R e \approx 140$, therefore inducing a destabilizing effect by promoting free vortices at lower values of $V R$. Also note the existence of small lip vortices on the downstream corners for $R e=202$, an expected consequence of flow inertia in the flow in a re-entrant corner.

The effect of $R e$ on the critical value of the $V R$ for the onset of central vortices is shown in figure 15. It is clear that inertia prompts the transition to secondary flow to occur at lower values of $V R$. Even though inertia accelerates the appearance of free vortices, we should note that for low $V R($ eg. $V R=10)$ free vortex formation is not 


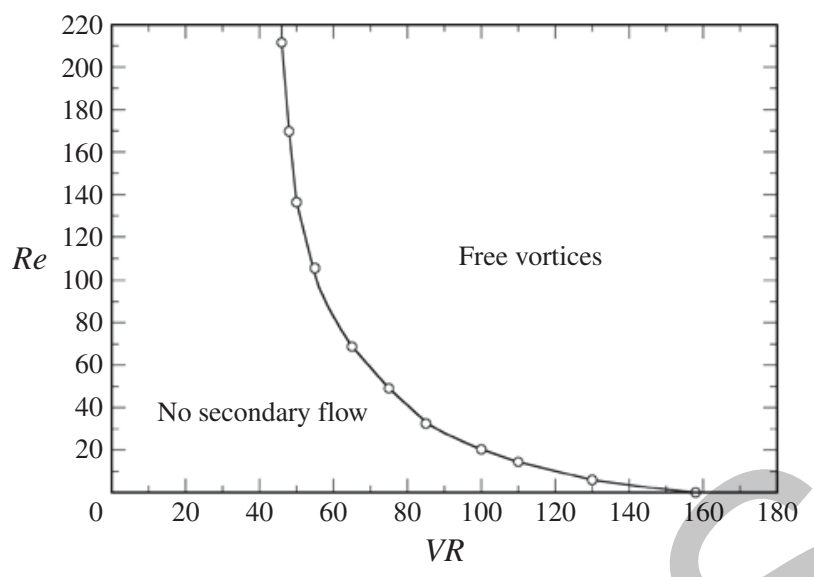

FIGURE 15. Stability map: effect of $R e$ on the critical velocity ratio for the onset of free vortices ( $2 \mathrm{D}$ flow, $W R=1$ ).

observed for the whole range of Reynolds numbers tested $(0 \leqslant R e \lesssim 210)$, while for high $V R($ e.g. $V R=1000)$ free vortices are always present even under creeping flow conditions, as shown in figure 3.

We should emphasize that in the case of inertial flows, it is not possible to use the superposition principle described in $\S 4.1$ for creeping flows and the linear combination of two independent results is no longer a solution of the Navier-Stokes equation due to the presence of the nonlinear advective term; in these cases, the critical $R e$ was determined numerically for each $V R$.

\section{Three-dimensional results}

In this section we compare the results obtained experimentally in a microfluidic channel of constant depth, as described in $\S 2$, and those obtained numerically in the corresponding three-dimensional geometry (with top and bottom bounding walls) under equivalent flow conditions.

Figure 16 shows the flow patterns observed maintaining $Q_{1}$ constant while varying $Q_{2}$ (and therefore varying $V R$ and $R e$ simultaneously). The grey-scale images were acquired at the channel centre plane using streak photography and the red lines are centre plane streamlines predicted numerically. Recirculations are also observed in this case, but its open three-dimensional structure is clearly distinct from the closed recirculations obtained in the numerical calculations for two-dimensional creeping flow shown in $\S 4$. Good agreement is obtained between experimental observations and three-dimensional numerical predictions, both in terms of the critical conditions for the appearance of recirculations and in terms of the flow patterns.

In figure 17(a), we show a three-dimensional view of the flow-focusing device with representative pathlines obtained numerically to highlight the three-dimensionality of the flow. This example corresponds to that shown in figure $16(d)$, and we can see that the fluid enters the recirculation at the centre plane, rotates towards its eye and exits the recirculation moving towards the top/bottom bounding walls. Evidence for this three-dimensionality is also observed experimentally (figure 17b) by injecting a dyed solution (using Rhodamine B) as described in $\S 2$. Using this technique we can observe that most of the fluid entering through the central mainstream channel (dyed 
(a)

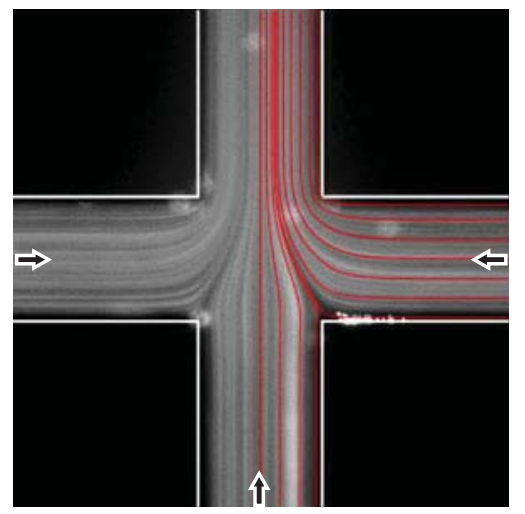

(c)

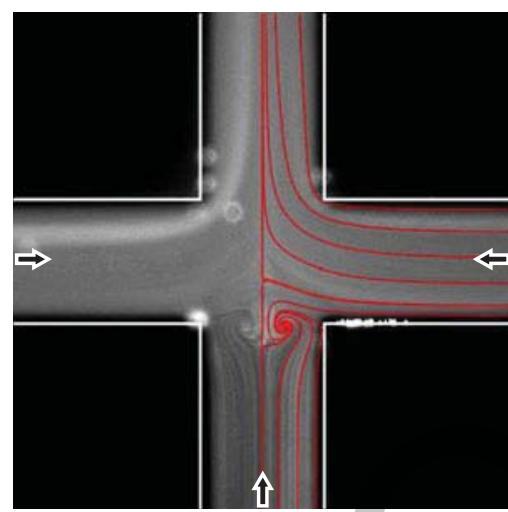

(b)

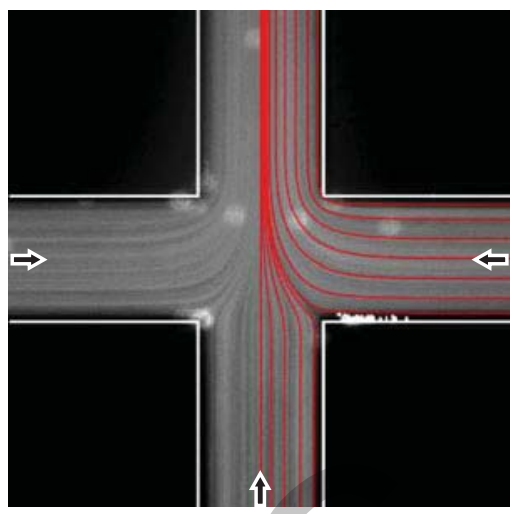

$(d)$

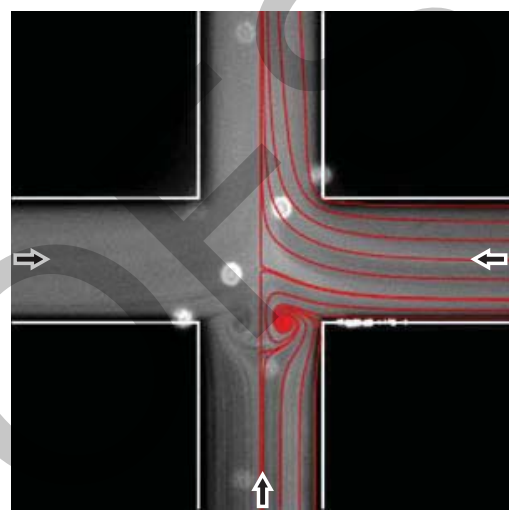

FIGURE 16. (Colour online) Flow patterns in the 3D flow-focusing geometry for varying $V R$ and $R e$. Comparison between experimental (grey-scale images) and numerical pathlines (red lines) at the centre plane. (a) $Q_{1}=0.3 \mathrm{ml} \mathrm{h}^{-1}, Q_{2}=0.3 \mathrm{ml} \mathrm{h}^{-1}, V R=1, R e=2.8 ;$ (b) $Q_{1}=$ $0.3 \mathrm{ml} \mathrm{h}^{-1}, Q_{2}=0.9 \mathrm{ml} \mathrm{h}^{-1}, V R=3, R e=6.5 ;(c) Q_{1}=0.3 \mathrm{ml} \mathrm{h}^{-1}, Q_{2}=15 \mathrm{ml} \mathrm{h}^{-1}$, $V R=50, R e=94.2 ;(d) Q_{1}=0.3 \mathrm{ml} \mathrm{h}^{-1}, Q_{2}=18 \mathrm{ml} \mathrm{h}^{-1}, V R=60, R e=113$.

with Rhodamine B) exits the device close to the sidewalls and at a plane that is closer to the bounding walls, where the fluorescence of the Rhodamine B dye can still be perceived, albeit with a lower intensity than in the central entrance channel. Indeed, the dyed region in the exit channel matches closely the projection of the three-dimensional numerical pathlines (see figure 17b).

In figure 18 we show a flow classification map in the $V R-R e$ parameter space. For each set of flow conditions $(V R, R e)$, we determined whether or not vortices were present and this is identified by different symbols. Each thin solid line corresponds to numerical simulations obtained for a constant value of $Q_{1}$ (and varying $Q_{2}$ ). From this map it is clear that inertia has a destabilizing effect, inducing the appearance of recirculations at lower critical $V R$ than under creeping flow conditions. In the limit of $V R=0$, no steady central vortices are seen in the range of Re tested. On the contrary, the bounding walls have a stabilizing effect for low $R e$ flow conditions, delaying the onset of the central vortices (compare the critical value of $V R$ under creeping flow conditions for the three-dimensional case $(V R \approx 628)$ with that for the two-dimensional case presented in $\S 4.1(V R \approx 159))$. Interestingly, at high $R e$ the opposite trend is found and the bounding walls actually have a destabilizing effect, as is clear from a 
(a)

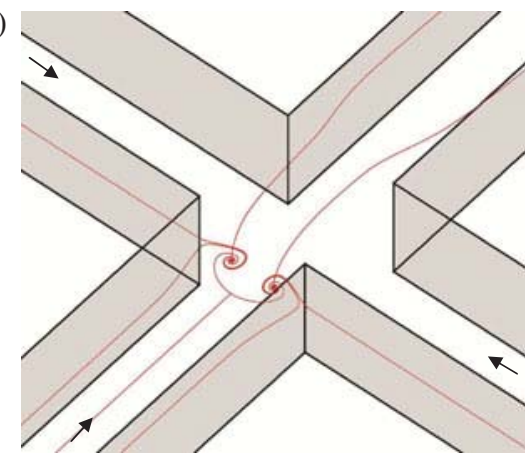

(b)

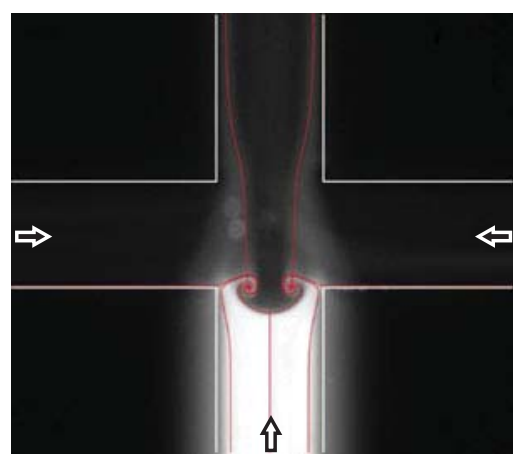

FIgURE 17. (Colour online) The 3D nature of the flow (VR=60, $R e=113, Q_{1}=0.3 \mathrm{ml} \mathrm{h}^{-1}$, $Q_{2}=18 \mathrm{ml} \mathrm{h}^{-1}$ ). (a) Three-dimensional view of the flow-focusing device with representative streamlines obtained numerically (in red) showing that the flow is highly three-dimensional with the fluid entering the recirculation near the centre plane, rotating towards its eye and exiting close to the top/bottom bounding walls. (b) Comparison between the experimental photograph (grey-scale image) at the central plane obtained when Rhodamine is injected in the main central channel and a projection of the numerical streamlines (in red).

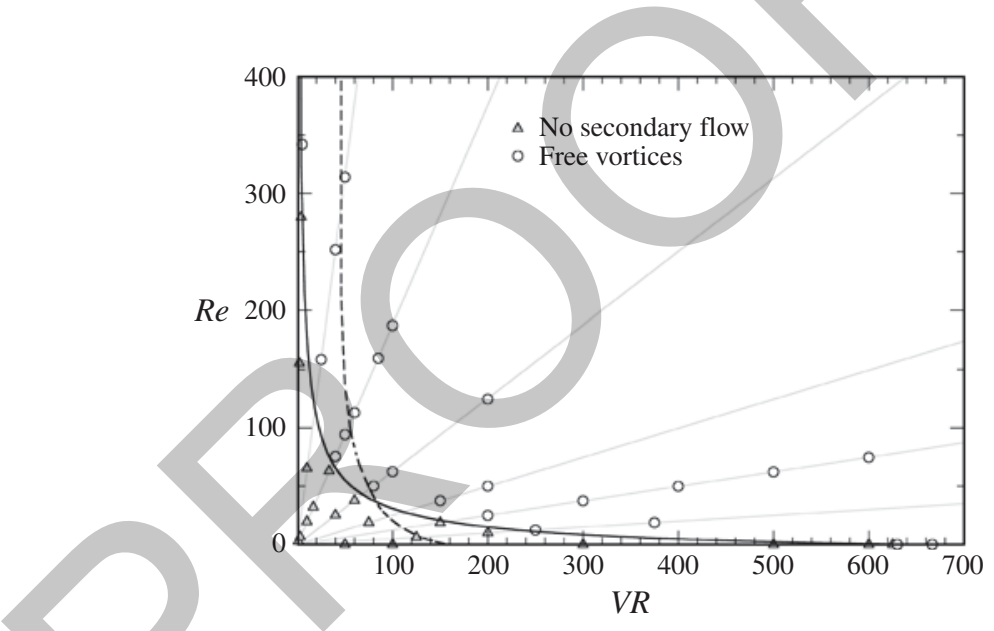

FIGURE 18. Flow classification map in the $V R-R e$ domain (3D flow, $W R=1$ ). The thick solid line highlights the critical values for the onset of free vortices. The dashed line is that of figure 15 for $2 \mathrm{D}$ flow.

comparison between the solid and dashed lines in figure 18, marking the transition for the three-dimensional and two-dimensional geometries, respectively.

\section{Conclusions}

We performed a systematic numerical and experimental study on Newtonian fluid flow through microfluidic devices in which hydrodynamic flow focusing is produced using two balanced lateral sheath streams that shape a third inlet stream. In particular, we focus on the onset and enhancement of symmetrical wall-detached recirculations that form near the centreline above a critical value of the ratio of inlet average velocities. Two- and three-dimensional numerical calculations were performed using 
a finite volume method, and comparison of the three-dimensional results with the experimental flow visualizations shows very good agreement.

We demonstrate that central vortices arise above a critical velocity ratio and are preceded by the onset of diverging streamlines despite the hydrodynamic focusing imposed by the lateral sheath streams. Recirculation growth was observed as $V R$ was increased, but was shown to be limited by the formation of a second recirculation of lower intensity upstream of the primary one. The effect of the width ratio was studied numerically, and it was shown that the critical $V R$ above which the recirculations are observed depends non-monotonically on this geometrical parameter. In addition, we were able to show that vortex formation occurs even under creeping flow conditions, despite flow inertia enhancing its appearance. In contrast, the presence of the walls in three-dimensional geometries has a stabilizing effect at low Re, delaying the onset of these recirculations, which are no longer closed recirculations.

Most importantly, we show for this complex flow the implications of Stokes flow theory, since under creeping flow conditions we are able to predict the flow field for any $V R$ by combining linearly the results corresponding to any two other independent solutions (e.g. $V R=0$ and $V R \rightarrow \infty$ ) in the same geometry. This approach allows us to explain why vortices form upstream of the central region near the vertical centreline and also to predict the critical value of $V R$ for the onset of central vortices without having to perform additional experiments or numerical calculations under creeping flow conditions.

\section{Acknowledgements}

The authors acknowledge the funding support provided by Fundação para a Ciência e a Tecnologia (FCT), COMPETE and FEDER through projects PTDC/EMEMFE/099109/2008， PTDC/EME-MFE/114322/2009， PTDC/EQU-FTT/118716/2010, REEQ/928/EME/2005.

\section{REFERENCES}

Alves, M. A., OliveirA, P. J. \& Pinho, F. T. 2003 A convergent and universally bounded interpolation scheme for the treatment of advection. J. Non-Newtonian Fluid Mech. 41, $47-75$.

Alves, M. A. \& Poole, R. J. 2007 Divergent flow in contractions. J. Non-Newtonian Fluid Mech. 144, 140-148.

Anna, S. L., Bontoux, N. \& Stone, H. A. 2003 Formation of dispersions using 'flow focusing' in microchannels. Appl. Phys. Lett. 82, 364-366.

AnNA, S. L. \& MAYER, H. C. 2006 Microscale tipstreaming in a microfluidic flow. Phys. Fluids 18, 121512.

Arratia, P. E., Gollub, J. P. \& Durian, D. J. 2008 Polymeric filament thinning and breakup in microchannels. Phys. Rev. E 77, 036309.

Bird, R. B., Armstrong, R. C. \& Hassager, O. 1987 Dynamics of Polymeric Liquids, vol. 1: Fluid Dynamics. Wiley.

Boger, D. V. \& Binnington, R. J. 1990 Circular entry flows of fluid M1. J. Non-Newtonian Fluid Mech. 35, 339-360.

Campo-Deaño, L., Galindo-Rosales, F. J., Pinho, F. T., Alves, M. A. \& Oliveira, M. S. N. 2011 Flow of low viscosity Boger fluids through a microfluidic hyperbolic contraction. J. Non-Newtonian Fluid Mech. 166, 1286-1296.

Chinang, T. P., Sheu, T. W. H. \& WANG, S. K. 2000 Side wall effects on the structure of laminar flow over a plane-symmetric sudden expansion. Comp. Fluids 29, 467-492. 
Dendukuri, D. \& Doyle, P. S. 2009 The synthesis and assembly of polymeric microparticles using microfluidics. Adv. Mater. 21, 1-16.

Escudier, M. 1988 Vortex breakdown: observations and explanations. Prog. Aerosp. Sci. 25, 189-229.

Evans, R. E. \& WALters, K. 1989 Further remarks on the lip-vortex mechanism of vortex enhancement in planar-contraction flows. J. Non-Newtonian Fluid Mech. 32, 95-105.

Garstecki, P., Stone, H. A. \& Whitesides, G. M. 2005 Mechanism for flowrate controlled breakup in confined geometries: a route to monodisperse emulsions. Phys. Rev. Lett. 94, 164501 .

Hulsen, M. A. 1993 Numerical simulation of the divergent flow regime in a circular contraction flow of a viscoelastic fluid. Theor. Comput. Fluid Dyn. 5, 33-48.

James, D. F., Chandler, G. M. \& Armour, S. J. 1990 A converging channel rheometer for the measurement of extensional viscosity. J. Non-Newtonian Fluid Mech. 35, 421-443.

JENSEN, K. 1998 Chemical kinetics: smaller, faster chemistry. Nature 393, 735-737.

Knight, J. B., Vishwanath, A., Brody, J. P. \& Austin, R. H. 1998 Hydrodynamic focusing on a silicon chip: mixing nanoliters in microseconds. Phys. Rev. Lett. 80, 3863-3866.

Leibovich, S. 1978 The structure of vortex breakdown. Annu. Rev. Fluid Mech. 10, 221-246.

LucCA-Negro, O. \& O’Doherty, T. 2001 Vortex breakdown: a review. Prog. Energy Combust. Sci. 27, 431-481.

LUO, W.-J. 2009 Effect of ionic concentration on electrokinetic instability in a cross-shaped microchannel. Microfluid. Nanofluid. 6, 189-202.

Mcdonald, J. C., Dufy, D. C., Anderson, J. R., Chiu, D. T., Wu, H. \& Whitesides, G. M. 2000 Fabrication of microfluidic systems in poly (dimethylsiloxane). Electrophoresis 21, 27-40.

Meinhart, C. D., Wereley, S. T. \& Gray, M. H. B. 2000 Volume illumination for two-dimensional particle image velocimetry. Meas. Sci. Technol. 11, 809-814.

Moffatt, H. K. 1964 Viscous and resistive eddies near a sharp corner. J. Fluid Mech. 18, 1-18.

Nie, Z., Seo, M., Xu, S., Lewis, P. C., Mok, M., Kumacheva, E., Whitesides, G. M., Garstecki, P. \& Stone, H. A. 2008 Emulsification in a microfluidic flow-focusing device: effect of the viscosities of the liquids. Microfluid. Nanofluid. 5, 585-594.

Nisis AKO, T., TORII, T. \& HiguCHI, T. 2004 Novel microreactors for functional polymer beads. Chem. Engng J. 101, 23-29.

OlIVEIRA, P. J. 2003 Asymmetric flows of viscoelastic fluids in symmetric planar expansion geometries. J. Non-Newtonian Fluid Mech. 114, 33-63.

Oliveira, M. S. N., Alves, M. A., McKinley, G. H. \& Pinho, F. T. 2007 Viscous flow through microfabricated hyperbolic contraction. Exp. Fluids 43, 437-451.

OliveirA, P. J. \& Pinho, F. T. 1999 Numerical procedure for the computation of fluid flow with arbitrary stress-strain relationships. Numer. Heat Transfer B 35, 295-315.

Oliveira, P. J., Pinho, F. T. \& Pinto, G. A. 1998 Numerical simulation of nonlinear elastic flows with a general collocated finite-volume method. J. Non-Newtonian Fluid Mech. 79, 1-43.

Oliveira, M. S. N., Pinho, F. T., Poole, R. J., Oliveira, P. J. \& Alves, M. A. 2009 Purely elastic flow asymmetries in flow-focusing devices. J. Non-Newtonian Fluid Mech. 160, 31-39.

Oliveira, M. S. N., Rodd, L. E., McKinley, G. H. \& Alves, M. A. 2008 Simulations of extensional flow in microrheometric devices. Microfluid. Nanofluid. 5, 809-826.

Phan-Thien, N. \& TANneR, R. I. 1977 A new constitutive equation derived from network theory. J. Non-Newtonian Fluid Mech. 2, 353-365.

Rodd, L. E., Scott, T. P., Boger, D. V., Cooper-White, J. J. \& McKinley, G. H. 2005 The inertio-elastic planar entry flow of low-viscosity elastic fluids in micro-fabricated geometries. J. Non-Newtonian Fluid Mech. 129, 1-22.

Soulages, J., Oliveira, M. S. N., Sousa, P. C., Alves, M. A. \& MCKinley, G. H. 2009 Investigating the stability of viscoelastic stagnation flows in T-shaped microchannels. J. Non-Newtonian Fluid Mech. 163, 9-24. 
Sousa, P. C., Coelho, P. M., Oliveira, M. S. N. \& Alves, M. A. 2011 Laminar flow in three-dimensional square-square expansions. J. Non-Newtonian Fluid Mech. 166, 1033-1048.

Townsend, P. \& Walters, K. 1994 Expansion flows of non-Newtonian liquids. Chem. Engng Sci. 49, 749-763.

Tsai, C.-H., Chen, H.-T., WAng, Y.-N., Lin, C.-H. \& FU, L.-M. 2006 Capabilities and limitations of two-dimensional and three-dimensional numerical methods in modelling the fluid flow in sudden expansion microchannels. Microfluid. Nanofluid. 3, 13-18. 